\title{
L'anthropologie et l'histoire naturelle de l'homme en 1800. Les enjeux d'un héritage
}

Jean-Luc Chappey

\section{(2) OpenEdition \\ Journals}

Édition électronique

URL : https://journals.openedition.org/ahrf/142

DOI : $10.4000 /$ ahrf.142

ISSN : 1952-403X

\section{Éditeur :}

Armand Colin, Société des études robespierristes

Édition imprimée

Date de publication : 1 juin 2000

Pagination : 47-54

ISSN : 0003-4436

\section{Référence électronique}

Jean-Luc Chappey, «L'anthropologie et l'histoire naturelle de l'homme en 1800. Les enjeux d'un héritage », Annales historiques de la Révolution française [En ligne], 320 | avril-juin 2000, mis en ligne le 23 janvier 2006, consulté le 23 avril 2022. URL : http://journals.openedition.org/ahrf/142 ; DOI : https://doi.org/10.4000/ahrf.142

\section{Ce document a été généré automatiquement le 23 avril 2022}

Tous droits réservés 


\title{
L'anthropologie et l'histoire naturelle de l'homme en 1800. Les enjeux d'un héritage
}

\author{
Jean-Luc Chappey
}

1 Au point de départ de ces réflexions se situe mon travail sur la Société des Observateurs de l'homme ${ }^{1}$ dont le personnel se regroupe à partir de décembre 1800 autour d'un ambitieux projet scientifique la construction d'une Anthropologie, c'est-à-dire d'une connaissance de l'homme considéré comme objet physique, moral et intellectuel. Rassemblant des représentants des savoirs les plus divers, la Société devient, pendant près de quatre ans, le théâtre de recherches menées dans des directions et sur des objets très différents de l'étude des facultés intellectuelles de l'homme et des conditions de leur progrès, menée autour de l'observation du jeune sauvage de l'Aveyron et du Chinois Tchong-A-Sam, à l'étude comparée des crânes, des langues et des mœurs, la science générale de l'homme des Observateurs a pour objectif de réunir des domaines de savoir, des pratiques et des modes de production intellectuelles qui sont, à l'époque, dispersés dans plusieurs institutions savantes comme le Muséum d'histoire naturelle, l'École de Médecine, l'Institut des sourds-muets ou l'École spéciale des Langues orientales. On trouve là, à l'évidence, une tension entre, d'une part, le projet anthropologique des Observateurs conçu comme un cadre possible d'homogénéisation des différents savoirs sur l'homme, et d'autre part, la dispersion institutionnelle des savoirs, dispersion illustrée au sein même des grandes institutions savantes par les divisions établies entre les chaires d'enseignement.

2 C'est pour dépasser cette tension que le 18 messidor an IX (9 juillet 1801), le secrétaire perpétuel de la Société, Louis-François Jauffret, présente devant les membres de la Société des Observateurs, son mémoire connu depuis sous le titre d'» Introduction aux mémoires de la Société des Observateurs de l'homme ». Ce discours a pour fonction de rendre cohérents les divers travaux présentés, depuis plus d'un an, par les membres la Société. S'appuyant sur les mémoires précédents, lus par Degérando, Cuvier et Moreau de la Sarthe à l'occasion de la préparation théorique de l'expédition du capitaine 
Baudin et sur les autres mémoires, récits de voyage, observations sur les sourds-muets et sur les aliénés présentés à l'occasion de la première séance publique organisée par la Société en thermidor an VIII, Jauffret y précise les différents domaines de savoirs qui doivent composer l'Anthropologie. Dans un effort de mise en ordre, il tente d'en construire une synthèse épistémologique et méthodologique. Il entreprend, par ailleurs, de construire un réel projet commun en établissant des objets de recherches collectifs autour desquels doivent se réunir tous les membres de la Société des Observateurs de l'homme.

3 L'enjeu de ce texte est donc crucial pour les Observateurs. D'une part, alors que la Société n'a publié ni de liste de son personnel ni de recueil de ses mémoires, Jauffret doit construire une identité visible et commune au groupe des Observateurs et à son projet anthropologique. En ce sens, on peut dire que ce texte constitue la marque la plus évidente d'une tentative disciplinaire ou d'une institutionnalisation du savoir anthropologique autour de normes épistémologiques et méthodologiques appelés à devenir les règles du groupe des Observateurs.

4 C'est dans cette perspective que, dans son discours, Jauffret propose de « renfermer [l'Anthropologie] dans certaines bornes ». Précisant que l'Anthropologie doit réunir plusieurs sciences, telles que l'hygiène ou l'anatomie, Jauffret déclare que «la Société ne perdra jamais de vue que son but est de n'approfondir ces différentes sciences qu'en ce qui touche à l'histoire naturelle de l'homme proprement dite $»^{2}$. La question que je poserai est double tout d'abord, pourquoi Jauffret place-t-il l'Anthropologie des Observateurs sous l'égide de "l'histoire naturelle de l'homme "? Ensuite, et cette seconde question se rattache directement à la première, qu'entend Jauffret lorsqu'il parle « d'histoire naturelle de l'homme » Certes, on pourrait alléguer que l'équivalence synonymique établie par Jauffret entre l'Anthropologie et «l'histoire naturelle de l'homme » n'est pas originale en 1800. Le jeune médecin François Péron l'a posée avant lui, en prairial anVIII (juin 1800), dans le titre de son mémoire présenté devant les professeurs de l'École de Médecine, «Observations sur l'anthropologie, ou l'histoire naturelle de l'homme ", mémoire dans lequel Péron se propose de rechercher les causes de la santé "presque inaltérable $»^{3}$ des populations sauvages à travers l'étude des diverses influences physiques et morales auxquelles ces populations sont soumises. Par ailleurs, Pierre-Jean-Georges, dans ses Rapports du physique et du moral qui constitue alors la référence essentielle de la communauté médicale, emploie de nombreuses fois la notion « d'histoire naturelle de l'homme " pour désigner cette étude générale de la nature humaine constituée de deux «points de vue » indissociables, le physique et le moral, science autour de laquelle il appelle la communauté savante à se regrouper ${ }^{4}$. Lorsqu'il place l'Anthropologie des Observateurs dans les "bornes" de "l'histoire naturelle de l'homme ", Jauffret se serait-il seulement conformé à un "air du temps » Je ne le pense pas. Dans notre travail, nous avons fait l'hypothèse que cette appropriation de "l'histoire naturelle de l'homme " pour définir l'Anthropologie des Observateurs correspond chez Jauffret à des intérêts, à des prises de position, qui renvoient, non seulement à la nature singulière du projet anthropologique des Observateurs, mais aussi à la composition particulièrement hybride du groupe des Observateurs. Je voudrais ainsi revenir sur les usages possibles de ce recours à "l'histoire naturelle de l'homme» chez les Observateurs de l'homme. Pour ce faire, il faut prendre en compte différentes échelles d'analyse et tenter de relier une histoire 
théorique de l'Anthropologie des Observateurs, à une histoire sociale, voire politique et religieuse, du personnel qui compose la Société.

5 Le premier enjeu, le plus évident parce souligné par Jauffret lui-même, est un enjeu institutionnel. En plaçant l'Anthropologie sous la bannière de « l'histoire naturelle de l'homme ", Jauffret, porte-parole des Observateurs, cherche à démarquer la Société et son pro jet scientifique des autres sociétés savantes et de leurs projets scientifiques. Nous sommes là en présence d'une stratégie de distinction qu'on peut qualifier aussi de stratégie publicitaire. Alors que la Société est présidée par le célèbre botaniste AntoineLaurent Jussieu, il s'agit pour ses membres d'affirmer l'originalité de leur projet anthropologique au sein d'une communauté savante où d'autres institutions de savoir se réclament également de la construction d'une science générale de l'homme. Dans son discours, Jauffret justifie ainsi le recours à "l'histoire naturelle de l'homme » en affirmant que "cette direction particulière... offrira [à la Société] les recherches les plus neuves, les plus importantes, et aura l'avantage de ne pas confondre ses travaux avec ceux des Sociétés spéciales de médecine et de chirurgie $»^{5}$. Jauffret fait sans doute allusion ici à la Société médicale d'émulation fondée en l'an VI par des élèves de l'École de Médecine, Alibert, Bichat ou Moreau de la Sarthe, qui se regroupent autour de la construction d'une science générale de l'homme fondée sur les principes constitutifs de la «médecine philosophique » ou "médecine morale », science dont Cabanis a tracé la voie dans ses différents mémoires lus à l'Institut National à partir de l'an $\mathrm{IV}^{6}$. À travers son discours, Jauffret affirme clairement que le personnel et le projet anthropologique des Observateurs ne peuvent être confondus avec ceux de cette société savante. La Société des Observateurs, contrairement à ce que l'on a pu croire ${ }^{7}$, n'est pas constituée uniquement de médecins, ni même de naturalistes. Aux côtés de ces groupes, on trouve des représentants de savoirs, tels que les antiquaires ou les orientalistes, qui sont appelés à jouer un rôle tout aussi important dans la construction de l'Anthropologie.

6 En se démarquant ainsi nettement des sociétés savantes que l'on peut qualifier de "sociétés spécialisées", Jauffret affirme donc que l'Anthro-pologie ne saurait se réduire à un domaine de savoir particulier telle que l'hygiène ou l'anatomie comparée. Tous les savoirs qui ont comme objet l'homme considéré dans ses dimensions physiques et morales sont ainsi appelés à contribuer à part égale à la construction de l'Anthropologie. Contrairement à Cabanis et aux médecins qui s'en réclament, pour qui la science générale de l'homme doit être fondée sur la physiologie, contrairement également à certains naturalistes qui, comme Cuvier, entendent faire de l'anatomie comparée le support de l'étude de l'homme, Jauffret indique que l'Anthropologie des Observateurs ne saurait, en aucun cas, se réduire ou s'appuyer sur un domaine de connaissances particulier dont les représentants pourraient prétendre à un statut privilégié. En affirmant que l'Anthropologie est le produit de la réunion des représentants de savoirs les plus divers, Jauffret légitime la nature particulièrement hétérogène du personnel de la Société et en assure l'équilibre institutionnel.

7 Mais que nous dit cette affirmation quant au statut de cette nature humaine dont les Observateurs revendiquent l'étude Il me semble qu'en refusant de réduire l'Anthropologie à un domaine de connaissance particulier, et plus précisément en refusant de considérer, à l'instar de Cabanis et de ses émules, que la connaissance de l'organisation physiologique doit servir de support et rendre possible les progrès de la connaissance du moral de l'homme, Jauffret affirme qu'il est impossible d'admettre que le moral et le physique doivent être considérés comme deux substances indissociables. 
Si Jauffret, au nom des Observateurs, présente, comme l'un des objets essentiels de l'Anthropologie, l'étude des rapports entre le moral et le physique, il défend dans son discours-programme de l'an IX une conception dualiste de la nature humaine, en opposition à la théorie moniste défendue par Cabanis. Jauffret a-t-il lu Cabanis et connaît-il réellement la complexité théorique de sa pensée Je ne le sais pas. Mais il me paraît indéniable que c'est contre lui et contre ce qu'il considère comme une pensée matérialiste, accusée de provoquer le malheur et le désespoir de l'homme, que Jauffret porte de très vives attaques. À ce titre, le discours-programme dont on peut reconnaître la portée disciplinaire, est aussi un discours pamphlétaire et polémique dirigé contre tous les savants qui prétendre réduire le moral au physique et, par là, peuvent mettre en cause un principe présenté comme essentiel dans l'Anthropologie des Observateurs, à savoir la distance absolue entre l'homme et l'animal.

C'est dans cette perspective que j'ai tenté de montrer, et cela peut être contesté, qu'il était possible de retrouver dans l'Anthropologie des Observateurs un écho, un héritage de l'histoire naturelle de l'homme de Buffon. Certes, il ne s'agit pas pour moi de dire qu'il existe une filiation parfaite ou « naturelle » entre Buffon et les Observateurs, mais de montrer comment Buffon et certains principes épistémologiques de son Histoire naturelle de I'homme ont pu être instrumentalisés par les Observateurs. Comme toute entreprise de construction d'héritage, l'appropriation par les Observateurs de l'histoire naturelle de l'homme de Buffon est faite de distorsions, de simplifications, de travestissements, voire de trahisons. Ce qu'il m'importe donc de voir, c'est comment une certaine lecture de l'histoire naturelle de Buffon a pu être utilisée par les Observateurs pour répondre à des attentes spécifiques. Pour aller vite, j'évoquerai deux aspects qui me semblent significatifs de cet usage de l'héritage de Buffon par les Observateurs. Selon moi, «l'histoire naturelle de l'homme» de Buffon a servi, en particulier à travers la récupération par les Observateurs du principe de l'homo duplex, d'instrument théorique susceptible de démarquer, voire d'opposer, leur Anthropologie à la science de l'homme physiologiste de Cabanis et, plus généralement, à des théories qui, comme le transformisme, pouvaient en partager certains fondements. Contrairement aux interprétations canoniques, la Société des Observateurs ne peut pas être considérée comme une succursale de l'Idéologie ${ }^{8}$. Elle regroupe en effet, avec un noyau très actif de catholiques, certains de ses adversaires les plus virulents ; à leurs côtés, on trouve ceux qui, comme Joseph-Marie Degérando et Pierre Laromiguière, " compagnons de route de l'Idéologie », prennent, à partir de l'an IX, nettement leur distance avec elle. Néanmoins, la Société des Observateurs ne peut pas être simplement considérée comme un cadre de contre-offensive théorique, politique et religieuse, contre les principes de la science de l'homme de Cabanis et de Destutt de Tracy, dont elle accueille certains de ses partisans les plus actifs, tels que Pinel et Moreau de la Sarthe.

La Société reste avant tout un cadre scientifique au sein duquel les Observateurs, audelà de leurs divergences, tentent effectivement de trouver les assises d'un travail en commun. Dès lors, nous pouvons nous demander si, en plaçant leur projet anthropologique sous l'égide de l'histoire naturelle de Buffon, les Observateurs ne trouvent pas là un moyen de maintenir un consensus, une unanimité possible entre les différents membres de la Société dont les positions scientifiques, religieuses et politiques sont particulièrement hétérogènes, voire opposées. Une semaine avant la signature du Concordat, Buffon et son histoire naturelle de l'homme seraient peut-être, pour les Observateurs, les seules références possibles à partir desquelles adversaires et 
partisans des Lumières, catholiques et médecins philosophes, pourraient encore travailler ensemble à la construction de l'Anthropologie. Instrument de distinction, voire pour certains d'opposition, à l'Idéologie, support d'un « équilibre des tensions " au sein du groupe des Observateurs, voilà pour moi, deux raisons qui justifient la présence de Buffon chez les Observateurs en 1801.

J'achèverai cette communication par une réflexion sur les usages sociaux et sociologiques de cet héritage de l'histoire naturelle de l'homme de Buffon chez les Observateurs. Ce dernier point me permettra d'inscrire mes réflexions dans une perspective plus large concernant la "transition des savoirs » que l'on peut constater entre 1800 et 1810. Les usages sociaux de l'héritage buffonien ont déjà été analysés dans de nombreux travaux. Je pense particulièrement à ceux de P. Duris ${ }^{9}$ et de P. Corsi ${ }^{10}$. En résumé, ces travaux montrent comment certains naturalistes, doublement victimes du succès du groupe des linnéens, d'une part, et de la sédentarisation du savoir, d'autre part, qui fait passer les voyageurs-naturalistes du statut de producteurs à celui de simples collecteurs du savoir naturaliste, ont pu s'appuyer sur la revendication de l'héritage de Buffon pour défendre et tenter de maintenir leur position sociale au sein de la communauté savante. J'ai pour ma part constater qu'on assiste au phénomène identique chez les Observateurs auxquels appartiennent d'ailleurs certains de ces naturalistes " héritiers de Buffon ", tels que Sonnini de Manoncourt ou Patrin. Comme toutes les sociétés savantes parisiennes de l'époque, la Société des Observateurs est composée de figures savantes éminentes, mais aussi de personnages secondaires, des "seconds couteaux », qui, voyageurs, vulgarisateurs, pédagogues pour enfants comme Jauffret, ou bibliothécaires, n'occupent pas une position d'autorité dans l'espace savant du Consulat. C'est d'ailleurs parmi ces derniers que l'on trouve les réels animateurs de la Société et de son projet anthropologique. Or, se pose à eux un problème de légitimité scientifique: comment maintenir leur statut de savant face aux professeurs, aux membres des grandes institutions de savoir, qui contrôlent les matériaux et les normes de productivité des savoirs Si on les trouve parmi les animateurs les plus actifs de la Société, c'est que, pour eux, l'Anthropologie doit être un moyen de défendre leur position au sein de l'espace savant. C'est dans cette perspective qu'il me parait possible de parler d'un usage social de l'histoire naturelle de l'homme de Buffon chez les Observateurs. Je pense en effet que Buffon et son histoire naturelle de l'homme ont pu être utilisés comme des contre-modèles aux pratiques sociales de productivité du savoir qui fragilisent le statut des « seconds couteaux » à partir de 1800 , je veux parler des phénomènes de spécialisation méthodologique et de professionnalisation, qui constituent deux faits majeurs de l'évolution du monde savant entre 1800 et 1810 .

11 Au moment où il prononce son discours devant les Observateurs, Jauffret organise depuis prairial an IX des "promenades pédagogiques", promenades qui sont présentées comme une tentative de faire passer l'Anthropologie de la théorie à la pratique. Au-delà de leur contenu, ces promenades nous mettent en présence d'une certaine vision de la science, une science " utile", accessible au public et susceptible d'être construite et diffusée, en dehors des institutions de savoir et par des individus ne bénéficiant pas d'une solide légitimité scientifique et institutionnelle. Au cours des promenades de Jauffret, on pratique une Anthropologie, très éloignée des formes positives que l'on a voulu lui donner. Il s'agit plus d'une "science littéraire » à visée encyclopédique où sont valorisés le "beau langage ", la curiosité et le pittoresque qui distraient le public tout en l'instruisant. Par là, ces promenades, comme le Dictionnaire d'histoire naturelle dirigé par Sonnini de Manoncourt, apparaissent 
bien comme le signe d'une résistance face à la science qui, confisquée par un corps de spécialistes, s'enferme dans les institutions de savoir.

Si Jauffret place l'Anthropologie des Observateurs sous l'égide de l'histoire naturelle de l'homme de Buffon, c'est justement pour qu'elle ne devienne pas l'apanage des corps de spécialistes, mais pour qu'elle conserve une fonction d'utilité et de vulgarisation. L'héritage de Buffon et de son histoire naturelle de l'homme servirait ainsi, pour Jauffret et, plus largement, pour tous les acteurs secondaires de l'espace savant, de supports à l'expression d'une nostalgie d'un ordre social de la science qui tend progressivement à disparaître entre 1800 et 1810, période pendant laquelle ces acteurs secondaires sont progressivement marginalisés ou exclus de l'espace savant. La Société des Observateurs et son projet anthropologique ne résisteront pas à cette crispation sociale de l'espace savant qui entraîne leur disparition en 1804. L'histoire naturelle de l'homme de Buffon est victime de cette même crispation sociale dans l'ordre de production et de diffusion du savoir naturaliste. En dépit du succès éditorial remporté de manière très éphémère par le Dictionnaire d'histoire naturelle rédigé par les " héritiers de Buffon », Cuvier signe en 1808, dans son Rapport à l'Empereur, l'acte de décès de l'héritage buffonien. Néanmoins, contrairement aux Observateurs et à leur Anthropologie, Buffon et son histoire naturelle ne disparaissent pas complètement. Ils sont réappropriés par les membres d'une société particulière, la Société d'Agriculture, qui n'est pas une société savante, mais une société d'experts, au sein de laquelle Buffon, rejeté hors des cadres des sciences naturelles spéculatives, devient le modèle référentiel de l'histoire naturelle « utile».

\section{NOTES}

1.Jean-Luc Chappey, "La Société des Observateurs de l'homme (1799-1804). Genèse, personnel et activités d'une société savante sous le Consulat ", thèse d'histoire dirigée par Catherine Duprat, Université de Paris I, I.H.R.F., 1999, 3 vol.

2.Louis-François Jauffret, "Introduction aux mémoires de la Société des Observateurs de l'homme ", dans J. Copans, J. Jamin, Aux Origines de l'Anthropologie française, Paris, Le Sycomore, 1978, p.73.

3.François Péron (F.), “Observations sur l'anthropologie, ou l'histoire naturelle de l'homme, la nécessité de s'occuper de l'avancement de cette science, et l'importance de l'admission sur la flotte du capitaine Baudin d'un ou plusieurs naturalistes, spécialement chargés des recherches à faire sur ce sujet », dans J.Copans, J.Jamin, op.cit., p. 182.

4."Le principe des sciences morales, et par conséquent ces sciences elles-mêmes, rentreraient dans le domaine de la physique ; elles ne seraient plus qu'une branche de l'histoire naturelle de l'homme... D'après la direction que suit depuis trente ans la direction de l'esprit humain, les sciences physiques et naturelles semblent avoir généralement obtenu le premier pas. Leurs rapides progrès, dans un si court espace de temps, ont rendu l'époque actuelle la plus brillante de leur histoire : et c'est en rapprochant d'elles... toutes les autres sciences et tous les arts qu'on peut espérer, avec 
fondement, de les voir tous éclairés enfin d'un jour en quelque sorte égal », cité dans P.J.G. Cabanis, Rapports de physique et du moral [1802], Euvres philosophiques de Cabanis, édition établie et annotée par Cl. Lehec et J. Cazeneuve, Paris, P.U.F., 1956, t. I, p. 110. 5.Louis-François Jauffret, "Introduction aux mémoires de la Société des Observateurs de l'homme ", op.cit., p. 73.

6.Voir Jean-François Alibert, "Discours sur les rapports de la médecine avec les sciences physiques et morales », Mémoires de la Société d'émulation médicale, Paris, Fuchs, an VI (1798), vol. 2, pp.1-112.

7.M. Bouteiller, "La Société des Observateurs de l'homme, ancêtre de la Société d'anthropologie de Paris ", Bulletins et mémoires de la Société d'anthropologie de Paris, 1956, Xe série, pp. 448-465.

8.Contrairement à ce qu'affirme Marc Régaldo, Un Milieu intellectuel. La Décade philosophique, 1794-1807, Service de reproduction des thèses, Lille III, Paris, H. Champion, 1976, t. 2, p. 641, note 1.

9.Pascal Duris, Linné et la France 1780-1850, Genève, Droz, 1993, 283 p.

10.Pietro Corsi, “ Buffon sous la Révolution et l'Empire », in J. Gayon (dir.), Buffon 88.

Actes du colloque international, Paris, Vrin, 1992, pp. 639-648.

\section{RÉSUMÉS}

Dans leur tentative pour créer les conditions d'une institutionnalisation et d'une légitimation de leur programme scientifique, les membres de la Société des Observateurs de l'homme (1799-1804) ont recours à l'héritage de Buffon, dont l'Histoire naturelle de l'homme peut être considérée comme la référence centrale, épistémologique et méthodologique, de leur Anthropologie. Cette appropriation de l'héritage buffonien peut surprendre, car le statut scientifique de Buffon fait l'objet de nombreuses contestations dans la communauté naturaliste des années 1800. Dès lors, il convient de s'interroger sur les motivations et les enjeux de cette appropriation. Le recours à l'héritage de Buffon peut être interprété à la lumière de stratégies sociales menées par des « seconds couteaux » de l'espace savant, et nous nous interrogerons sur le rôle de cet héritage dans une possible offensive menée par les Observateurs contre les principes épistémologiques de l'Idéologie de Cabanis et de Tracy. Ces réflexions permettent de proposer une série d'hypothèses sur la disparition des Observateurs en 1804 et sur «l'échec» de leur programme anthropologique, lequel ne résiste pas aux transformations profondes qui caractérisent les modes sociaux, épistémologiques, méthodologiques de la production et de la diffusion des savoirs entre 1800 et 1810 .

\section{Anthropology and the natural history of man in 1800 : What's at stake in a legacy.}

In an attempt to create the right conditions for the institutionalization and legitimation of their scientific programme, the members of the Société des Observateurs de l'homme (1799-1804) appealed to the legacy of Buffon, whose Natural History of Man can be considered as the central epistemological and methodological reference for their Anthropology. This appropriation of Buffon's legacy might appear surprising, as his scientific standing was the object of a number of attacks within the community of natural history during the 1800s. Thus, we need to investigate the stakes at play in this appropriation, as well as the motivations for it. The appeal to Buffon's 
legacy can be interpreted in the light of the social strategies of the «minor figures» in the field, and we examine the role played by this legacy in a possible offensive led by the Observateurs against the epistemological principles of the Idéologues Cabanis and de Tracy. The resulting observations will allow us to propose a series of hypotheses concerning the disappearance of the Observateurs in 1804 , as well as the «failure» of their anthropological programme, which did not survive the profound transformations that characterized the social, epistemic and methodological forms of production and diffusion of knowledge between 1800 and 1810.

INDEX

Mots-clés : anthropologie, Société des Observateurs de l'homme, idéologues, Buffon, Cabanis

\section{AUTEUR}

JEAN-LUC CHAPPEY

ATER à l'Université de Reims, Institut d'histoire de la Révolution française (IHRF) 